\title{
PROPOSTA EDUCATIVA PARA O AVANÇO NAS ESCOLHAS ALIMENTARES DE ALÉRGICOS EM SERVIÇOS DE ALIMENTAÇÃO: RELATO DE PROJETO
}

Rita de Cassia de Souza FERNANDES ${ }^{1}$

Marina de Almeida LIMA ${ }^{2}$

Noeli Aparecida Rosa de MORAIS ${ }^{3}$

Daniela Maria Alves CHAUD ${ }^{4}$

\begin{abstract}
${ }^{1}$ Graduanda do curso de Nutrição do Centro de Ciências Biológicas e da Saúde da Universidade Presbiteriana Mackenzie. E-mail: ritadecsfernandes@gmail.com

${ }^{2}$ Graduanda do curso de Nutrição do Centro de Ciências Biológicas e da Saúde da Universidade Presbiteriana Mackenzie. E-mail: manutrimack@gmail.com

${ }^{3}$ Graduanda do curso de Nutrição do Centro de Ciências Biológicas e da Saúde da Universidade Presbiteriana Mackenzie. E-mail: noeli.rosa@gmail.com

${ }^{4}$ Nutricionista, mestre e doutora, professora do curso de Nutrição do Centro de Ciências Biológicas e da Saúde da Universidade Presbiteriana Mackenzie. E-mail: daniela.chaud@mackenzie.br
\end{abstract}

Recebido em: 28/12/2016 - Aprovado em: 05/05/2017 - Disponibilizado em: 01/07/2017

\begin{abstract}
RESUMO
Objetivo: Fornecer subsídios para informar, educar e auxiliar os responsáveis pelos serviços de alimentação comerciais quanto à alergia alimentar e suas consequências na ocorrência de uma ingestão acidental, de modo a permitir uma escolha alimentar segura desse grupo altamente vulnerável. Métodos: Elaboração de folder, vídeo explicativo e cardápio modelo com base no manual português "Alergia Alimentar para a Restauração", para serem apresentados em reuniões agendadas nos sindicatos da área ou no próprio restaurante. Desenvolvimento: Percebendo as repercussões biopsicossociais envolvidas e a necessidade de um novo posicionamento frente a esse cenário, buscou-se, por meio dos recursos elaborados, alertar os responsáveis pelos serviços de alimentação quanto à gravidade dos sintomas e apresentar os principais ingredientes potencialmente alergênicos (the big eight), para que, então, passassem a expor para os seus clientes nos cardápios os alérgenos contidos nas preparações, visto que seriam instruídos a identificar os ingredientes utilizados por meio das fichas técnicas e da leitura dos rótulos alimentares, alem de adaptarem o serviço para evitar contaminações cruzadas e promoverem um atendimento responsivo pelos funcionários do estabelecimento. Conclusão: Apesar de ser uma proposta ainda a ser viabilizada, trata-se de um avanço social para escolhas alimentares de alérgicos em serviços de alimentação, sendo necessário ainda avaliar a aplicabilidade dos itens pelos responsáveis para que seja garantido o Direito do Consumidor. Também é proposta a disseminação em redes sociais dos materiais educativos desenvolvidos e de uma hashtag específica para conseguir maior visibilidade.

Palavras-chave: Alérgenos. Hipersensibilidade Alimentar. Rotulagem de Alimentos. Serviços de Alimentação. Socialização.
\end{abstract}

\section{EDUCATION PROPOSAL FOR ADVANCING FOOD CHOICES OF}

\section{ALLERGIC IN FOOD SERVICES: PROJECT REPORT}

\footnotetext{
ABSTRACT

Objective: To provide subsidies for informing, educating and assisting commercial food service providers about food allergy and its consequences in the event of accidental ingestion, so as to allow a safe food choice for this highly vulnerable group. Methods: Elaboration of a folder, explanatory video and model menu based on the portuguese manual 
"Food Allergy for Restoration", to be presented in meetings scheduled in the area's trade unions or in the restaurant itself. Development: Realizing the biopsychosocial repercussions involved and the need for a new positioning in front of this scenario, it was sought, through the elaborated resources, to alert those in charge of food services about the severity of the symptoms and to present the main potentially allergenic ingredients (the big eight), so that they could then expose the allergens in their preparations to their customers on the menus, since they would be instructed to identify the ingredients used though the technical data sheets and the reading of the food labels, in addition to adapting the service to avoid cross-contamination and to promote responsive service for the establishment's employees. Conclusion: Although it is a proposal still to be made feasible, it is a social advance for food allergy choices in food services, and it is still necessary to evaluate the applicability of the items by those responsible to ensure consumer rights. It is so proposed the dissemination in social networks of the education materials developed and a specific hashtag to achieve greater visibility.

Keywords: Allergens. Food Hypersensitivity. Food Labeling. Food Services. Socialization.

\section{INTRODUÇÃO}

Apesar de a maioria das pessoas usufruírem de grande diversidade na alimentação, sendo esta importante por suprir as necessidades nutricionais e sensoriais, indivíduos com alergias alimentares podem ter sua saúde em risco se ingeridos alguns ingredientes, mesmo em pequenas quantidades, devido a reações adversas graves (BRASIL, 2014; BRASIL, 2006; TEIXEIRA, 2010).

As alergias alimentares são definidas como uma hipersensibilidade do organismo humano a determinadas proteínas que são reconhecidas erroneamente como antígenos após ingestão, inalação ou mesmo toque a alimentos ou aditivos alimentares (PEREIRA; MOURA, CONSTANT, 2008; REY; SILVESTRE, 2009). Envolvem manifestações cutâneas (urticária, inchaço, coceira e eczema), gastrointestinais (diarreia, dor abdominal, refluxo, vômito), respiratórias (rinoconjuntivite, tosse, rouquidão, chiado no peito) e a anafilaxia, que atinge diversos órgãos e sistemas de maneira simultânea, sendo esta a considerada mais grave
(BATISTA; FREITAS; HAACK， 2009; PROTESTE; PÕE NO RÓTULO, 2014).

Sabe-se que a alergia alimentar é um problema de saúde pública que vem crescendo nos países industrializados. De acordo com Burks, James e Eigenmann (2011), é estimado que um quarto da população mundial irá enfrentar uma reação adversa a alimento durante a vida. Quanto à epidemiologia das alergias alimentares, estima-se que apesar de aproximadamente 2,5\% da população adulta ser afetada, sua ocorrência é mais comum nos primeiros anos de vida, afetando 6,0\% das crianças abaixo dos três anos de idade (BATISTA; FREITAS; HAACK, 2009; PEREIRA; MOURA; CONSTANT, 2008).

Visto que o principal tratamento consiste na eliminação do alimento alergênico, evitar a exposição dos principais ingredientes é essencial para se reduzirem os sintomas decorrentes, proporcionando assim uma melhor qualidade de vida aos indivíduos acometidos. No que se refere à anafilaxia, estimativas internacionais indicam que entre 
30,0 a 50,0\% dos casos são causadas por alimentos. Os alérgicos e suas famílias, portanto, buscam evitar o consumo de ingredientes alergênicos, adotando estratégias como a leitura cuidadosa dos rótulos (ANVISA, 2016). Porém, tal tarefa tornou-se árdua devido à necessidade atual da alimentação fora do lar derivada da falta de tempo para o preparo e consumo dos alimentos, uma vez que muitos alérgenos estão presentes em alimentos amplamente utilizados na culinária, como leite, ovos e trigo (COCCO et al., 2007; SAMPSON, 2004; SOLÉ et al., 2012).

Observa-se que os estabelecimentos comerciais não alertam previamente a composição dos alimentos oferecidos, o que vai em desacordo com os direitos básicos do consumidor previstos pelo artigo $6^{\circ}$ da Lei $n^{\circ}$ 8.078, sendo obrigatória a informação adequada e clara sobre produtos e serviços oferecidos, bem como seus eventuais riscos (BRASIL, 1990). É inegável a necessidade atual da identificação dos ingredientes potencialmente alergênicos contidos em preparações, principalmente devido ao aumento da sintomatologia e epidemiologia da alergia alimentar, assim como após vigência da obrigatoriedade da disponibilização de alérgenos contidos em alimentos industrializados nos rótulos depois de ter ocorrido intensa manifestação da sociedade (ANVISA, 2016).
Ademais, a contaminação cruzada dos alimentos também pode desencadear uma reação alérgica, devido ao uso de equipamentos compartilhados, partículas dispersas no ar, limpeza, transporte, estoque e manipuladores envolvidos na fabricação dos produtos e preparações (PROSTESTE; PÕE NO RÓTULO, 2014).

O tratamento e a prevenção da alergia alimentar só terão sucesso com abordagem multidisciplinar, estando à indústria de alimentos e os serviços de alimentação (como restaurantes e lanchonetes), responsáveis por elaborar e implementar ferramentas que permitam auxiliar no processo de trabalho e de gestão de tarefas, no ensejo de minimizar o risco de exposições acidentais e, assim, promover a qualidade de vida daqueles que possuem alergia alimentar, uma vez que a alimentar-se fora do lar configura-se como uma atividade de socialização, tanto para a classe trabalhadora, quanto para àqueles que realizam encontros entre amigos e passeios familiares (CAVALCANTE et al., 2015; PADUA et al., 2016).

Tendo em vista os aspectos mencionados, o projeto educativo proposto teve como objetivo fornecer subsídios para informar, educar e auxiliar os responsáveis pelos serviços de alimentação comerciais quanto à alergia alimentar e suas consequências na ocorrência de uma ingestão acidental, de modo a permitir uma escolha 
alimentar segura desse grupo altamente vulnerável e a garantia de socialização.

\section{METODOLOGIA}

Foi utilizado como base teórica para o projeto o manual português "Alergia Alimentar para a Restauração", que tem como premissas o Regulamento $\mathrm{n}^{\mathrm{o}} \quad 1169 / 2011$ publicado no Diário Oficial da União Europeia (PADUA et al., 2016),

Primeiramente, o projeto educativo visa orientar os responsáveis pelos serviços de alimentação para que eles compreendam e se conscientizem com relação à alergia alimentar, além de incentivar a prática de atitudes voltadas ao conhecimento de ingredientes potencialmente alergênicos nas preparações culinárias, bem como explicitar maneiras para se evitar uma contaminação cruzada.

Busca-se, de tal forma, que os responsáveis pelos serviços de alimentação promovam a exposição, nos cardápios, dos alérgenos alimentares contidos nas preparações que distribuem para que os frequentadores alérgicos pudessem realizar melhores escolhas alimentares sem o risco inerente de eventos associados.

Os recursos e materiais propostos, definidos como apropriados, envolveram a elaboração de um folder com o conteúdo sobre essa temática, conforme Figura 1, um vídeo explicativo e a elaboração de um cardápio modelo, após adaptação (PÕE NO RÓTULO, 2014).

Figura 1 - Folder elaborado para a conscientização de responsáveis de serviço de alimentação sobre alergia alimentar. São Paulo, 2016.

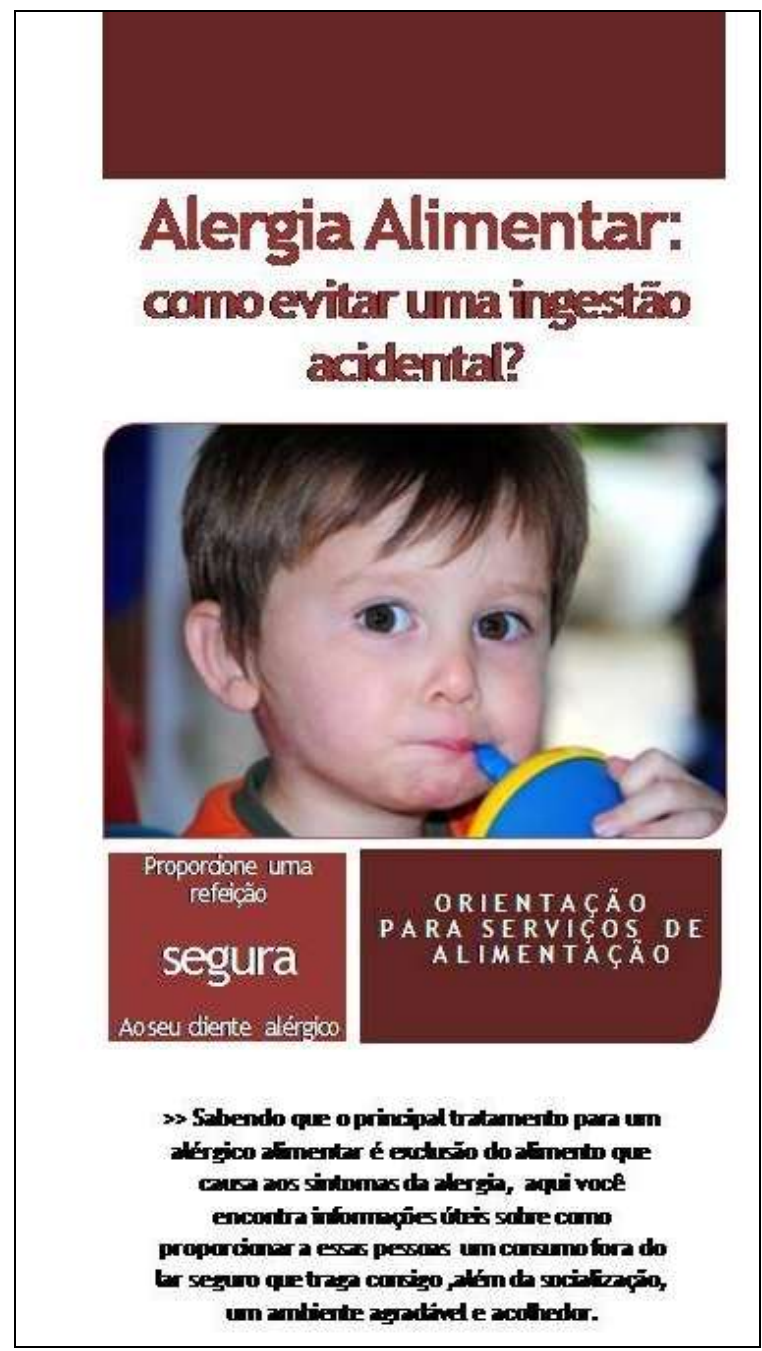

Cabe ressaltar que foram utilizadas imagens de domínio público para a confecção do folder.

Apesar de também existirem restaurantes institucionais, a principal estratégia foi a abordagem em serviços de alimentação comerciais, que incluem 
restaurantes, hotéis, padarias, pastelarias, bares, cafés e food trucks, visto que oferecem alimentos potencialmente alergênicos e possuem a disponibilização de cardápios aos clientes, bem como se configuram como principais locais procurados cotidianamente para a realização de refeições.

O plano de ação consiste em apresentar o vídeo explicativo, entregar folder com informações relevantes e exemplificar aplicabilidade com cardápio modelo, assim como sanar possíveis dúvidas de donos e responsáveis por meio de reuniões a serem realizadas nos próprios estabelecimentos ou nos sindicatos da área.

\section{DESENVOLVIMENTO}

A concepção da proposta educativa exposta no presente artigo se deu inicialmente por um trabalho exigido na disciplina de Educação Nutricional incluída na grade curricular do curso de Nutrição. A afeição das pesquisadoras ao assunto levou à escolha do tema, uma vez que buscaram ampliar os conhecimentos da alergia alimentar com bases científicas e, cuja percepção da necessidade de mudanças nos serviços de alimentação surgiu por meio de um debate após exibição do curta-metragem "Incondicional" no Centro Cultural Banco do Brasil em setembro de 2016, divulgado nas redes sociais, que envolveu especialistas, a atriz e cineasta responsável Aymara Limma e familiares de alérgicos alimentares.

Assim, percebendo as repercussões biopsicossociais envolvidas e a necessidade de um novo posicionamento frente a esse cenário, buscou-se elaborar um projeto educativo para que os responsáveis pelos serviços de alimentação pudessem perceber a gravidade dos sintomas, além de receberem orientação quanto aos principais ingredientes considerados potencialmente alergênicos, sendo estes denominados the big eight (leite, trigo, soja, ovos, peixes, crustáceos, amendoim e castanhas), cuja presença ou risco da presença em alimentos industrializados deve ser obrigatoriamente expressa nas embalagens por meio dos rótulos, conforme explicitado no folder elaborado conforme Figura 2 (ANVISA, 2016; REY; SILVESTRE, 2009). 
Figura 2 - Folder elaborado para a conscientização de responsáveis de serviço de alimentação sobre alergia alimentar. São Paulo, 2016.

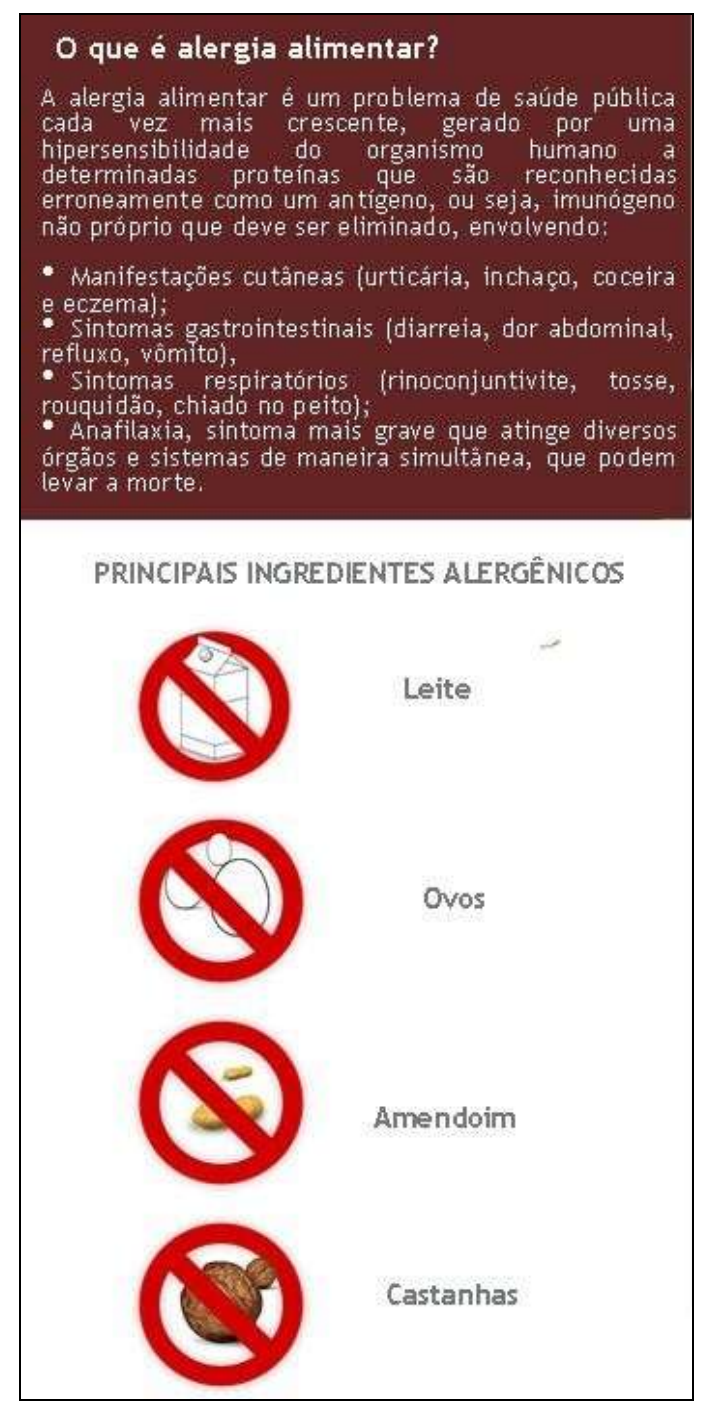

Visto a precaução de alérgicos alimentares quanto à ingestão acidental de um alergênico e a necessidade de socialização desses indivíduos, buscou-se por meio dos recursos elaborados na presente proposta educativa um novo posicionamento por parte dos responsáveis pelos serviços de alimentação quanto à identificação dos ingredientes utilizados nas preparações, como também a leitura cuidadosa do rótulo que devem resultar na exposição dos ingredientes potencialmente alergênicos aos seus clientes por meio dos cardápios, salvaguardando, dessa maneira, o Direito do Consumidor, tão crucial no exercício da cidadania, assim como ocorre nos alimentos embalados, regulamentados por meio da Resolução da Diretoria Colegiada (RDC) no 26 (ANVISA, 2016; BRASIL, 1990).

O cardápio modelo elaborado, exposto na Figura 3, se configura como um facilitador na exposição desses ingredientes alergênicos, dando liberdade aos donos e responsáveis pelos estabelecimentos de adequarem o layout e identidade visual do cardápio ao serviço.

Figura 3 - Cardápio modelo para serviços de alimentação com a disponibilização de alérgenos alimentares contidos nas preparações.

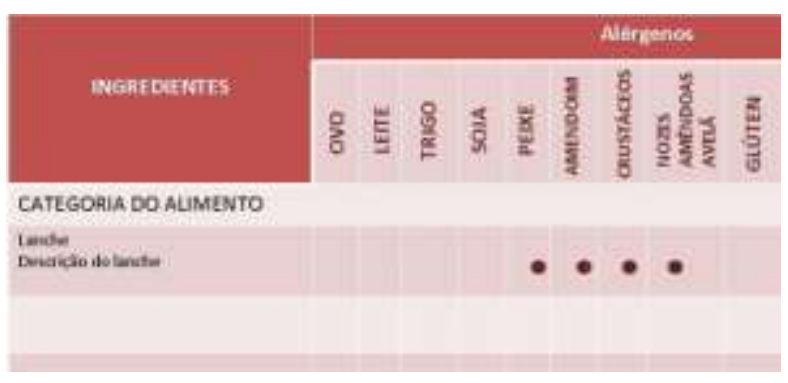

Assim, tendo o consumidor que possua alguma hipersensibilidade alimentar escolhido uma preparação que não contenha riscos destacados em cardápio ou pelo garçom, o responsável pelo estabelecimento deve lhe assegurar que o prato escolhido não conterá nenhum ingrediente que lhe cause dano devido a uma contaminação cruzada ou leitura inadequada de rótulo. 
Observa-se, nesse cenário, a importância das fichas técnicas, que, se concebidas de forma adequada, fornecem informações e instruções claras quanto aos produtos, equipamentos e utensílios utilizados no processo de elaboração dos alimentos nos estabelecimentos

comerciais

(VASCONCELOS;

CAVALCANTI;

BARBOSA, 2002).

Ademais, é válido reforçar que receitas ditas como não reveláveis devem expor, ao menos, a presença de alérgenos alimentares em sua composição, mesmo que em pequenas quantidades, assim como os auxiliares culinários (como a farinha para engrossar molhos), os usados para conferir sabor (manteigas nos grelhados) ou mesmo os incluídos nas marinadas (bebidas alcoólicas e especiarias) (PADUA et al., 2016).

É muito importante também não se esquecer da importância da higienização das mãos por parte dos manipuladores durante as diferentes etapas de preparação dos alimentos para se evitar uma possível contaminação cruzada de alérgenos (SOUZA et al., 2015).

Devido a possíveis contaminações cruzadas, é necessário todo um contexto de adaptação do serviço aos clientes, visto que os alimentos, os utensílios, o transporte das refeições e até mesmo as toalhas utilizadas podem conter alguns traços de alérgenos alimentares e causar a reação de hipersensibilidade advinda não somente da ingestão, mas também do toque ou mesmo da inalação (PADUA et al., 2016). Lembrandose que, de acordo com a Sociedade Brasileira de Pediatria e Associação Brasileira de Alergia e Imunologia (2008), em casos extremos, há risco considerável de morte desses indivíduos.

Não somente a garantia de um alimento livre de alérgenos contempla o quesito socialização; a forma de tratamento a ser dado às famílias ou aos clientes que possuem alergia alimentar pelos donos e garçons deve ter como premissa uma escuta acolhedora seguida da compreensão dos anseios e expectativas relatados, de modo a evitar o aparecimento de sintomas decorrentes da ingestão acidental (PADUA et al., 2016). Os garçons também devem ser instruídos no que se refere ao questionamento se, dentre os presentes, há algum alérgico alimentar, para que haja a exposição oral dos ingredientes potencialmente alergênicos contidos nas preparações, seguido da instrução de uma escolha, dentre as contidas no cardápio, mais apropriada.

Também foi inserido nos materiais educativos de que forma os colaboradores dos estabelecimentos comerciais podem atuar em caso de uma reação alérgica grave, visando uma busca imediata de ajuda médica por meio do Serviço de Atendimento Móvel de Urgência (SAMU). 
Por fim, cabe ressaltar que o vídeo elaborado já foi depositado no site de compartilhamento de vídeos YouTube pelas autoras (LIMA et al., 2016).

\section{CONCLUSÃO}

Foi desenvolvida uma proposta de avanço social para escolhas alimentares de alérgicos em serviços de alimentação, de modo a se prevenirem eventos associados à alergia alimentar, como a anafilaxia, por meio da garantia ao acesso facilitado a informações de alérgenos alimentares contidos nas preparações por meio de cardápios ou auxílio de garçons, além de adaptações no serviço para se evitarem contaminações cruzadas.

Salienta-se que se trata de uma proposta a ser viabilizada. É necessário que, além da aplicação dos materiais desenvolvidos, também haja uma avaliação constante das estratégias discutidas para se analisar se a proposta promoveu o efeito desejado por meio do acompanhamento desses serviços de alimentação, de modo a também garantir que os itens abordados sejam aplicados, dentre eles a explicitação dos alérgenos alimentares nos cardápios e o futuro treinamento de garçons e manipuladores.

Uma das estratégias, em curto prazo, será a disseminação em redes sociais dos materiais educativos desenvolvidos. Devido à repercussão promovida pela campanha "Põe no Rótulo”, o presente estudo visiona retomar tais debates em relação aos alérgenos alimentares presentes nos alimentos nas redes sociais. Para isso, propõe a utilização da hashtag adaptada para as novas necessidades da temática abordada “\#poenocardapio” para conseguir maior visibilidade por meio de multiplicadores que abraçam a causa.

Tal ação será avaliada e outras paulatinamente colocadas em prática, visando à melhoria da qualidade de vida desse grupo vulnerável.

\section{REFERÊNCIAS}

ANVISA. Agência Nacional de Vigilância Sanitária. Perguntas e Respostas sobre Rotulagem de Alimentos Alergênicos. Brasília/DF. $3^{a}$ ed. Jan. 2016. Disponível em: <http://portal.anvisa.gov.br/wps/wcm/connect lebf99e804b7a7680a847e82c3efbfd41/Pergun tas+e+Respostas+sobre+Rotulagem+de+Aler g\%C3\%AAnicos.pdf?MOD=AJPERES $>$.

Acesso em: 25 abr. 2016.

BATISTA, G.S.; FREITAS, A.M.F.; HAACK, A. Alergia alimentar e desmame precoce: uma revisão do ponto de vista nutricional. Com. Ciências Saúde. v.20, n.4, p.351-360, 2009.

BRASIL. Constituição Federal de. Lei n . 8.078, de 11 de setembro de 1990: Dispõe sobre a proteção do consumidor e dá outras providências. Diário Oficial da União. 1990.

BRASIL. Ministério da Saúde. Secretaria de Atenção à Saúde. Departamento de Atenção Básica.Guia alimentar para a população brasileira. 2 ed. Brasília: 2014.

BRASIL. Ministério da Saúde. Secretaria de Atenção à Saúde. Guia alimentar para a 
população brasileira: promovendo a alimentação saudável. 1 ed. Brasília: 2006.

BURKS, W.; JAMES, J.; EIGENMANN, P. Food allergy. 1 ed. US: Elsevier Health Sciences; 2011.

CAVALCANTE, C.M.S. Sentidos da alimentação fora do lar para homens idosos que moram sozinhos. Rev. Bras. Geriatr. Gerontol. v.18, n.3, 2015

COCCO, R.R. et al. Abordagem laboratorial no diagnóstico da alergia alimentar. Rev.

Paul. Pediatr., v. 25, n. 3, p. 258-265, 2007.

FOOD STANDARDS AGENCY. Guidance on Allergen Management and Consumer Information. 2016. Disponível em:

$<$ http://www.food.gov.uk/sites/default/files/m ultimedia/pdfs/maycontainguide.pdf $>$. Acesso em: 07 jun. 2015.

LIMA, M.A. et al. Alergia alimentar: proposta educativa para serviços de alimentação. Disponível em: $\langle$ https://youtu.be/1XCs0G_I7oY > Acesso em: 19 dez. 2016.

PADUA, I. et al. Alergia Alimentar na Restauração. Alergia Alimentar na Restauração. Disponível em:

$<$ http://nutrimento.pt/activeapp/wpcontent/uploads/2016/05/Alergia-Alimentarna-Restaura\%C3\%A7\%C3\%A3o.pdf>. Acesso em: 02 out. 2016.

PEREIRA, A.C.S.; MOURA, S. M.; CONSTANT, P. B. L. Alergia alimentar: sistema imunológico e principais alimentos envolvidos. Semina: Ciências Biológicas e da Saúde, Londrina, v.29, n.2, p. 189-200, jul./dez. 2008.

PÕE NO RÓTULO. Cardápio com a descrição dos alérgenos. 2014. Disponível em: $<$ https://www.facebook.com/poenorotulo/phot os/a.1408757802708338.1073741828.140842 1726075279/1454797524771032/?type=3 $>$.

Acesso em: 30 out. 2016.

PROSTESTE; PÕE NO RÓTULO. Cartilha de alergia alimentar. 2015. 20p. Disponível em:

$<$ http://poenorotulo.com.br/CartilhaAlergiaAl imentar_29AGO.pdf $>$. Acesso em: $13 \mathrm{abr}$. 2016.

REY, A.M.; SILVESTRE, A.A. Comer sem risco 2: As doenças transmitidas por alimentos.1 ed. São Paulo: Livraria Varela, 2009. 336p.

SAMPSON, H.A. Update on food allergy. Journal of Allergy and Clinical Immunology, v.113, n.5, p.805-819, 2004.

SOCIEDADE BRASILEIRA DE PEDIATRIA; ASSOCIAÇÃO BRASILEIRA DE ALERGIA E IMUNOLOGIA. Consenso brasileiro sobre alergia alimentar. Rev. Bras. Alerg. Imunopatol. v.31, n.2, 2008.

SOLÉ, D. et al. Guia prático de diagnóstico e tratamento da Alergia às Proteínas do Leite de Vaca mediada pela imunoglobulina E. Rev. Bras. Alerg. Imunopatol. v.35, n.6, 2012.

SOUZA, G.C. et al. Comida de rua: avaliação das condições higiênico-sanitárias de manipuladores de alimentos Ciência \& Saúde Coletiva, v.20, n.8, p.2329-2338, 2015.

TEIXEIRA, A.R.N. Alergias alimentares na infância. Porto: Universidade do Porto, 2010.

VASCONCELLOS, F.; CAVALCANTI, E.; BARBOSA, L. Menu: como montar um cardápio eficiente. São Paulo: Roca; 2002. 